\title{
WHI: RIESG OS DE CANCER MAMARIO Y PROBLEMAS CARDIOVASCULARES CON TERAPIA DE REEMPLAZO HORMONAL (THR) COMBINADA CONTINUA*
}

\author{
Moderador: $\quad$ Dra. Mercedes Ruiz F.1 \\ Participantes: Drs. Italo Campodónico G. ${ }^{2}$, Patricio Contreras C. $^{3}$, Raquel Herrera ${ }^{4}$, \\ Eugenio Arteaga H. ${ }^{5}$, Emiliano Soto R. ${ }^{1}$
}

Servicios y Departamentos de Obstetricia, Ginecología y Neonatología, Hospitales Clínico, ${ }^{1}$ San Borja-Arriarán. ${ }^{2}$ Salvador, ${ }^{3}$ Clínica Alemana, ${ }^{4}$ José Joaquín Aguirre, ${ }^{5}$ Departamento Endocrinología, Universidad Católica de Chile

Dra. M. Ruiz F.: La reciente difusión mediática de las evaluaciones del estudio (WHI) Women's Health iniciative, en relación a los riesgos de cáncer de mama y el incremento de efectos coronarios indeseables con el uso de la terapia de reemplazo hormonal en mujeres climatéricas ha provocado una voz de alerta y alarma a nivel mundial.

Consecuente con esta situación la Sociedad Chilena de Obstetricia y Ginecología, en conjunto con la Sociedad de Endocrinología y la Sociedad de Climaterio, ha estimado necesario escuchar las opiniones de sus miembros más destacados en esta área con el objeto de escuchar sus planteamientos.

La masiva asistencia de médicos especialistas y becados en esta Mesa Redonda es el mejor aval de la oportuna decisión del Directorio de nuestra Sociedad madre de Obstetricia y Ginecología para lograr esta convocatoria.

Solicito al Profesor Campodónico que inicie estas presentaciones relatándonos la historia de las THR en medicina.

Dr. I. Campodónico: A pesar de las décadas de evidencia acumulada de la observación, el balance de los riesgos y beneficios de la utilización de hormonoterapia sustitutiva en mujeres postmenopáusicas sanas permanece incierto.
La historia de la HTR en mujeres climatéricas comienza en los Estados Unidos a comienzos de la década de los cuarenta, como consecuencia del libro de Wilson "Femenine for ever" (Femenina para siempre) y la posibilidad de producir, a gran escala, estrógenos conjugados obtenidos de la orina de yeguas preñadas, activos por la vía oral.

Por su parte, también en Europa, comienza su amplia prescripción, al sintetizarse la molécula del 17 beta-estradiol, principal esteroide secretado por la gónada femenina.

Reconocidos y comprobados los efectos benéficos de la estrógeno terapia sobre las manifestaciones del síndrome climatérico, sobre la atrofia urogenital y la calidad de vida de las mujeres -la balanza se inclina fuertemente a su favor; primando el criterio coreado al unísono por mujeres, médicos y medios de comunicación: "¡Estrógenos para todas y por toda la vida!".

A poco andar diversos estudios observacionales otorgan al estrógeno terapia potenciales beneficios adicionales, de la mayor trascendencia para la salud de la mujer, entre otros: protección cardiovascular, protección ósea, sobre la memoria y los procesos cognoscitivos, sobre la piel, faneríos e inclusive la dentadura.

Todo marchaba "miel sobre hojuelas" y en las décadas de los cincuenta y de los sesenta las dis-

*Mesa Redonda realizada en la sesión del 6 de agosto de 2002 de la Sociedad Chilena de Obstetricia y Ginecología. 
crepancias se limitaban a si era mejor administrar los estrógenos en forma continua o en forma cíclica, con pausas terapéuticas.

Después de treinta años de uso indiscriminado de la estrógeno terapia, a comienzos de los años setenta, diversas publicaciones alertan sobre un aumento significativo del riesgo, entre las usuarias, de desarrollar hiperplasia y cáncer endometrial.

Cunde el pánico y como reacción visceral la estrógeno terapia es unánimemente execrada y proscrita: “¿Hormonas? ¡A ninguna mujer!”.

Otra vez la ley del péndulo: ¡por Dios que cuesta equilibrar la balanza!

Afortunadamente, como en casi todas las cosas de la vida, después de una mala suele venir una buena. La buena nueva fue que el agregado de progestinas permitía revertir los riesgos de hiperplasia y de cáncer endometrial asociados con la estrógeno-terapia no opuesta. Todo dependía, más que del tipo de progestina utilizada, de su dosis, considerándose críticos los días de administración.

El empleo combinado de estrógenos más progestinas dio origen al concepto de terapia de reemplazo hormonal. Se aconsejaba administrar los estrógenos sea en forma cíclica o continua, con el agregado de progestinas, durante 10-14 días por mes, de forma secuencial.

¡Cuanto más progestinas se administrara, tanto más eficaz la protección endometrial!

En el intertanto comenzó a postularse que la protección cardiovascular atribuida a los estrógenos no dependía tan solo, como se supuso inicialmente, de su efecto favorable sobre las lipoproteínas, sino que también merced a sus acciones sobre el endotelio vascular, efectos antioxidantes, inhibición de la formación de la placa de ateroma y muy en especial, por sus acciones vasodilatadores.

Recuerdo que un distinguido profesor visitante, buen amigo de muchos de nosotros, logró sorprendernos y casi entusiasmarnos al afirmar, que de acuerdo a su experiencia, en mujeres con crisis de angor pectoris, la administración sublingual de estrógenos conjugados exhibía efectos vasodilatadores coronarios, tanto o más eficaces que los clásicos nitritos utilizados tradicionalmente con tal objetivo. ¡Otra vez el péndulo oscilaba en un extremo límite!

Lamentablemente se demostró que las progestinas, tan útiles para la protección endometrial causaban efectos cardiovasculares negativos, al oponerse a buena parte de los efectos beneficiosos de los estrógenos. ¡Por tanto debían ser prescritas en dosis mínimas!

A fines de la década de los setenta algunos estudios epidemiológicos observacionales alertaron sobre un eventual aumento del riesgo relativo (RR) de cáncer de mama en usuarias de estrógeno terapia, el que se asociaba con el tipo de estrógeno utilizado, dosis, duración del tratamiento, edad de las mujeres, etc. Por otra parte, a nivel mamario, el agregado de progestinas parecía no tener el mismo efecto favorable protector observado a nivel endometrial e inclusive parecía incrementar el riesgo.

Como consecuencia de las terapias secuenciales combinando estrógenos y progestinas las usuarias continúan con sangrados vaginales, los que tienden a reaparecer al indicar HTR en mujeres con amenorrea instalada.

Un buen número de mujeres, en especial las más jóvenes, acepta de buen grado la persistencia de las menstruaciones, considerándolas como símbolos en feminidad, de eterna juventud, e incluye, como certificación de la eficacia de la terapia.

Se indicaba con pompa: ¡HTR para toda la vida!: madres y abuelas debían continuar menstruando junto a sus hijas y nietas. Convencidas de los beneficios de la HTR, muchas mujeres mayores deseaban continuar utilizándolas, pero sin presentar sangrados.

Con este objetivo se desarrollan los esquemas combinados continuos: estrógenos más progestinas todos los días, sin pausas, con el objetivo de inducir atrofia endometrial y la subsiguiente amenorrea.

Esta modalidad de administración, si bien, parece conservar muchos de los efectos beneficiosos atribuidos a la estrógenoterapia exclusiva o a los esquemas secuenciales; sus reales efectos sobre el aparato cardiovascular y la glándula mamaria comienzan a ser cuestionados.

Cada vez nuestras pacientes tienen acceso a mayores fuentes de información y nos exigen aclarar sus dudas y temores en base a datos concretos y actualizados.

En nuestro deber aconsejarlas en forma fidedigna y compartir con ellas nuestras incertidumbres, teniendo como objetivo común fomentar su salud y calidad de vida. Al discutir con ellas los potenciales beneficios, riesgos y efectos colaterales de la HTR y ante la falta de datos definitivos; acostumbrábamos decirles, tal como los solíamos hacer también al concluir algunas de nuestras presentaciones, que debíamos esperar unos cuatro o cinco años para tener una respuesta definitiva, cuando dispusiéramos de los datos de diversos estudios clínicos prospectivos, randomizados, controlados contra placebo y con suficiente número de sujetos que se encontraban en desarrollo. 
Esta tarde nos hemos reunido para analizar los sorpresivos resultados de uno de estos estudios, denominado WHI y que fue suspendido prematuramente al comprobarse que los riesgos superaban a los beneficios esperados. Los datos han tenido amplia difusión en los medios de comunicación, causando gran revuelo y desazón tanto entre las usuarias como entre los médicos.

La situación actual es que: ¡El péndulo se salió totalmente de órbita!

Se trata de un ensayo clínico muy bien diseñado, cuyo objetivo era evaluar los riesgos y beneficios de un particular esquema terapéutico, el más utilizado en Norteamérica, en base a estrógenos conjugados equinos y acetato de medroxiprogesterona, administrados por vía oral en forma combinada continua. En los siguientes relatores corresponderá el análisis crítico de los datos disponibles.

Me cabe la duda si será posible extrapolar sus resultados para todas las otras múltiples alternativas terapéuticas disponibles. Diferentes estrógenos, diferentes progestinas, diferentes vías de administración, diferentes pautas terapéuticas, tibolona, raloxifeno, etc.

Para terminar, permítame parangonar la larga historia de la HTR, con la evolución de una enfermedad bipolar. Las mujeres, los médicos y también los medios de comunicación hemos transitado en un constante zigzagueo entre períodos de euforia y exaltación colectivas, interrumpidas por episodios de depresión, en los que todo se ve negro, sin esperanza alguna. Mención aparte para el reducido, pero agresivo grupo que me permito definir como paranoides: enemigos declarados de las hormonas, las temen, las odian, se sienten perseguidos y las culpan de todos los males.

No existe en la actualidad una HTR ideal, debemos esperar el desarrollo, por parte de la industria farmacéutica, de una "bala mágica", que concentre todos los beneficios y esté exenta de los riesgos asociados a las terapias convencionales actuales.

¡La esperanza es lo último que muere! Esperamos entonces, que en un futuro no muy lejano el fiel del la balanza alcance el adecuado equilibrio.

Dra. M. Ruiz F.: Agradezco con su completa información tan ilustrativa. Gracias. Tiene la palabra el Dr. Emiliano Soto.

Dr. E. Soto R.: La Sociedad Chilena de Obstetricia y Ginecología y la Sociedad Chilena de Climaterio me han invitado a participar en esta Mesa Redonda que analizará el reciente trabajo publicado en la revista Journal of the American Medical
Association (JAMA) sobre Riesgos y beneficios de Estrógeno más Progestina en mujeres postmenopáusicas sanas, principales resultados del estudio controlado y randomizado de Women's Health Initiative (1). Se me ha solicitado presentar un resumen de dicho trabajo. Tarea difícil de realizar por lo importante de los contenidos de la publicación señalada. Sin embargo, es necesario comenzar señalando que el diseño del presente trabajo se enmarca en un conjunto de proyectos llevados a cabo por Women's Health Initiative (WHI), dependiente del National Institute of Health (NIH) cuyo inicio fue en 1992 y cuyo término está programado para el año 2007. En este conjunto se incluyen trabajos clínicos que contemplan estrategias en prevención y control de algunas de las patologías de mayor morbilidad y mortalidad en mujeres postmenopáusicas, incluyendo cáncer, enfermedad cardiovascular y fracturas osteoporóticas. Ellas comprenden tres tipos de intervenciones (2):

1. Dieta baja en grasas, que hipotéticamente prevendría el cáncer de mama y secundariamente disminuiría el riesgo cardiovascular.

2. Tratamiento de Sustitución hormonal, que reduciría el riesgo de enfermedad coronaria y otras enfermedades cardiovasculares y secundariamente reduciría el riesgo de fractura de cadera y otras fracturas, con un efecto adverso probable de incremento en el riesgo de cáncer de mama, y

3. Suplemento de Calcio y Vitamina D, que hipotéticamente reduciría el riesgo de fractura de cadera y secundariamente el riesgo de otras fracturas como asimismo el riesgo de cáncer colorrectal.

Aquellas pacientes no incluidas en algunos de los tres estudios clínicos serían enroladas en estudios observacionales.

En la iniciativa de Terapia de sustitución hormonal se establecieron dos grupos, el primero en el cual se confrontó la terapia de estrógenos conjugados equinos $0,625 \mathrm{mg} /$ día con acetato de medroxiprogesterona $2,5 \mathrm{mg} / \mathrm{día}$ en forma combinada versus placebo; $y$ el segundo en el que se estudia la terapia de estrógenos conjugados equinos 0,625 $\mathrm{mg} / \mathrm{día}$ versus placebo.

El trabajo en referencia entrega los resultados preliminares del primer grupo de estudio, el cual fue detenido tempranamente ya que los riesgos sobrepasaron los beneficios al cabo de un seguimiento promedio de 5,2 años (rango entre 3,5 y 8,5 años). El estudio con uso de estrógenos solos versus placebo continúa y debería finalizar el año 2005 , con un período de seguimiento promedio de 8,5 años.

El reclutamiento de las voluntarias se realizó mediante avisos en la prensa o mediante comuni- 
cación telefónica a mujeres entre 50 y 79 años, con amenorreas de 6 meses en sujetos con más de 55 años, o 12 meses para mujeres entre 50 y 54 años, que tuvieran un domicilio estable en los años de estudio y que firmaran, por supuesto, un consentimiento informado para entrar al estudio. Entre las contraindicaciones para ser reclutada estaban cualquier condición que significara riesgo de muerte dentro de los tres siguientes años, cáncer de mama, otros cánceres dentro de los últimos diez años, excepto cánceres de piel no melanomas, hematocrito y/o recuento de plaquetas bajos, y condiciones que no aseguran un buen seguimiento como alcoholismo o demencia.

El estudio se realizó mediante randomización en doble ciego con dos grupos, uno en el cual las voluntarias recibieron una tableta conteniendo $0,625 \mathrm{mg}$ de estrógenos conjugados equinos más 2,5 $\mathrm{mg}$ de acetato de medroxiprogesterona por día, y el otro una tableta placebo. En un principio hubo pacientes con útero que fueron randomizadas para el estudio de estrógenos solos, pero al aparecer las evidencias de incremento de cáncer endometrial en pacientes tratadas con estrógenos sin progestágenos (3), estas voluntarias fueron enroladas en el grupo de pacientes que recibieron estrógenos equinos con medroxiprogesterona. Se realizó un contacto telefónico postrandomización y se evaluaron eventos clínicos cada seis meses y se planificó una visita clínica anual. El total de mujeres enroladas en este estudio y su seguimiento se muestran en la Figura 1.

El control consistió en la realización de mamografía y examen clínico mamario anuales, electro-

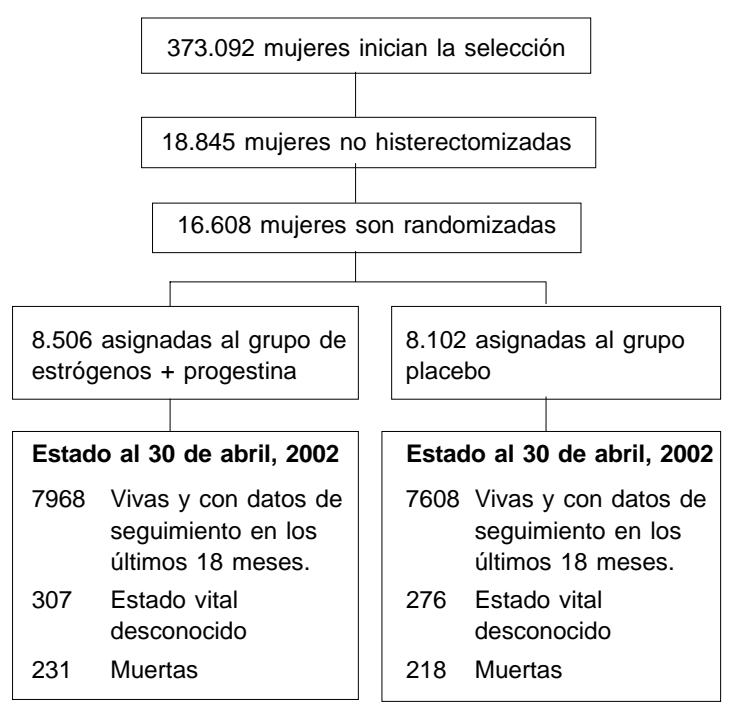

Figura 1. cardiograma basal y a los 3 y 5 años de tratamiento. Se discontinuaron aquellas pacientes que desarrollaron cáncer mamario, patología endometrial, melanoma maligno, meningioma, trombosis venosa profunda o embolia pulmonar, triglicéridos sobre $1.000 \mathrm{mg} / \mathrm{dl}$, o recibieron una prescripción de estrógenos, andrógenos o moduladores selectivos de receptores estrogénicos (SERM) por médico privado. La medicación fue suspendida temporalmente por infarto del miocardio, accidente vascular encefálico, fractura ósea, o cualquier lesión que requiriera hospitalización, cirugía con anestesia o inmovilización por más de una semana.

Los grupos no mostraron diferencias significativas entre ellos al analizar parámetros como edad, raza, uso de hormonas previo y tiempo de uso, índice de masa corporal, valores de presión arterial, hábito de fumar, paridad, edad al primer parto, tratamiento para diabetes, tratamiento para hipertensión arterial, niveles de colesterol, uso de estatinas, uso de aspirina, historia de infarto del miocardio, angina, accidente vascular encefálico, trombosis venosa profunda, embolismo pulmonar, pariente con cáncer mamario o fractura a edad mayor de 56 años. Sólo hubo diferencia significativa en el número de pacientes sometidas, previamente, a cirugía coronaria percutánea (120 en placebo v/s 96 en grupo con tratamiento).

Los resultados más importantes del estudio analizado se presentan en los cuadros siguientes y que se relacionan fundamentalmente con eventos cardiovasculares y con cáncer de mama invasor (Tabla I).

Las tendencias de estudios previos suponían resultados que el estudio demostró, ello en referencia a un incremento en los casos de trombosis venosa profunda, de embolismo pulmonar y de enfermedad tromboembólica en general. Llamativamente, los casos de cuadros coronarios, que se suponía disminuirían, aumentaron precozmente, siendo significativo el número de casos ocurrido en el grupo de paciente en tratamiento versus el grupo control desde el primer año de estudio. Por otra parte, los casos de fracturas disminuyeron como asimismo otros tipos de cánceres como el de endometrio y el colorrectal.

En el cuadro siguiente se presenta un resumen de los grupos de casos especiales relacionados con los accidentes cardiovasculares y con los casos de cáncer de mama. En el se relacionan los casos ocurridos relacionados con antecedentes previos (en los casos de accidentes cardiovasculares) y con el antecedente de uso y tiempo de terapia hormonal previa. En esta última situación 
Tabla I

\begin{tabular}{|c|c|c|c|c|}
\hline & $\begin{array}{l}E+P \\
(n=8506) \\
\text { número pacie }\end{array}$ & $\begin{array}{l}\text { clínicos I } \\
\text { Placebo } \\
\text { ( } n=8102) \\
\text { nualizados) }\end{array}$ & $H R$ & $95 \% \mathrm{Cl}$ \\
\hline Seguimiento (SD) & $62,2(16,1)$ & $61,2(15,0)$ & NA & NA \\
\hline $\begin{array}{l}\text { Enf. card. cor } \\
\text { Muerte ECC } \\
\text { IM no fatal } \\
\text { Revascularización }\end{array}$ & $\begin{array}{r}164(0,37) \\
33(0,07) \\
133(0,30) \\
183(0,42)\end{array}$ & $\begin{array}{r}122(0,30) \\
26(0,06) \\
96(0,23) \\
171(0,41)\end{array}$ & $\begin{array}{l}1,29 \\
1,18 \\
1,32 \\
1,04\end{array}$ & $\begin{array}{l}1,02-1,63 \\
0,70-1,97 \\
1,02-1,72 \\
0,84-1,28\end{array}$ \\
\hline $\begin{array}{l}\text { AVE } \\
\text { AVE fatal } \\
\text { AVE no fatal }\end{array}$ & $\begin{array}{r}127(0,29) \\
16(0,04) \\
94(0,21)\end{array}$ & $\begin{array}{l}85(0,21) \\
13(0,03) \\
59(0,14)\end{array}$ & $\begin{array}{l}1,41 \\
1,20 \\
1,50\end{array}$ & $\begin{array}{l}1,07-1,85 \\
0,58-2,50 \\
1,08-2,08\end{array}$ \\
\hline $\begin{array}{l}\text { Enf. tromb. emb. } \\
\text { Trombosis venosa profunda } \\
\text { Embolia pulmonar }\end{array}$ & $\begin{array}{r}151(0,34) \\
115(0,26) \\
70(0,16)\end{array}$ & $\begin{array}{l}67(0,16) \\
52(0,13) \\
31(0,08)\end{array}$ & $\begin{array}{l}2,11 \\
2,07 \\
2,13\end{array}$ & $\begin{array}{l}1,58-2,82 \\
1,49-2,87 \\
1,39-3,25\end{array}$ \\
\hline Enf. cardiovascular total & $694(1,57)$ & $546(1,32)$ & 1,22 & $1,09-1,36$ \\
\hline & $\begin{array}{l}E+P \\
(n=8506) \\
\text { número pacie }\end{array}$ & $\begin{array}{l}\text { clínicos II } \\
\text { Placebo } \\
\text { ( } n=8102) \\
\text { nualizados) }\end{array}$ & $H R$ & $95 \% \mathrm{Cl}$ \\
\hline $\begin{array}{l}\text { Cáncer total } \\
\text { Mama invasivo } \\
\text { Endometrial } \\
\text { Colorrectal }\end{array}$ & $\begin{array}{r}502(1,14) \\
166(0,38) \\
22(0,05) \\
45(0,10)\end{array}$ & $\begin{array}{r}458(1,11) \\
124(0,30) \\
25(0,06) \\
67(0,16)\end{array}$ & $\begin{array}{l}1,03 \\
1,26 \\
0,83 \\
0,63\end{array}$ & $\begin{array}{l}0,90-1,17 \\
1,00-1,59 \\
0,47-1,47 \\
0,43-0,92\end{array}$ \\
\hline Fractura total & $650(1,47)$ & $788(1,91)$ & 0,76 & $0,69-0,85$ \\
\hline $\begin{array}{l}\text { Cadera } \\
\text { Vertebral } \\
\text { Otras osteoporosis }\end{array}$ & $\begin{array}{r}44(0,10) \\
41(0,19) \\
579(1,31)\end{array}$ & $\begin{array}{r}62(0,15) \\
60(0,15) \\
701(1,70)\end{array}$ & $\begin{array}{l}0,66 \\
0,66 \\
0,77\end{array}$ & $\begin{array}{l}0,45-0,98 \\
0,44-0,98 \\
0,69-0,86\end{array}$ \\
\hline $\begin{array}{l}\text { Muerte otras causas } \\
\text { Muerte total }\end{array}$ & $\begin{array}{l}165(0,37) \\
231(0,52)\end{array}$ & $\begin{array}{l}166(0,40) \\
218(0,53)\end{array}$ & $\begin{array}{l}0,92 \\
0,98\end{array}$ & $\begin{array}{l}0,74-1,14 \\
0,82-1,18\end{array}$ \\
\hline Indice global & $751(1,70)$ & $623(1,51)$ & 1,15 & $1,03-1,28$ \\
\hline
\end{tabular}

resulta significativa la relación con el tiempo de uso de THR $(z=2,17)$ (Tabla II).

Aún cuando el estudio fue detenido por el número de casos de cáncer mamario invasivo, un número importante de eventos cardiovasculares habían sucedido para contraindicar la continuación de él hasta el tiempo planificado, pese a que la tendencia es a disminuir la diferencia entre eventos cardiovasculares de los grupos y placebo a medida que pasan los años. Lo determinado por este estudio WHI está en concordancia con otros estudios como el estudio HERS, con el estudio de reemplazo hormonal para ateroesclerosis y con el estudio en mujeres con angina inestable.

Referente al cáncer mamario, aún cuando existen evidencias previas en trabajos observacionales,
Tabla II

RESULTADOS CLINICOS (GRUPOS ESPECIALES)

\begin{tabular}{lcc}
\hline & $H R$ & Nominal 95\% Cl \\
\hline $\begin{array}{l}\text { ECV } \\
\text { ECC previa }+ \\
(\mathrm{n}=400, \text { seg }=57,4 \mathrm{~m})\end{array}$ & 1,28 & $0,64-2,6$ \\
ECC previa - & 1,28 & $1,00-1,65$ \\
Tro. ven. pr. + & 4,90 & $0,58-41,06$ \\
Tro. ven. pr. - & 2,06 & $1,54-2,76$ \\
AVE + & 0,46 & $0,05-4,51$ \\
Cáncer de mama & & \\
Nunca THR & 1,06 & $0,81-\quad 1,38$ \\
THR < 5a previos & 2,13 & $1,15-3,94$ \\
& & \\
THR 5-10a previos & 4,61 & $1,01-21,02$ \\
THR > 10a previos & 1,81 & $0,60-5,43$ \\
\hline
\end{tabular}


el trabajo analizado es el primer estudio randomizado que confirma que la terapia combinada de estrógenos más acetato de medroxiprogesterona aumenta el riesgo de esta patología y cuantifica la magnitud de dicho riesgo. El incremento significativo del riesgo se hace manifiesto a contar desde el cuarto año de terapia.

En forma resumida se puede establecer que por cada 100.000 mujeres que usen la terapia de estrógenos conjugados $0,625 \mathrm{mg} / \mathrm{día}$ asociada a acetato de medroxiprogesterona $2,5 \mathrm{mg} / \mathrm{día}$, en el período de un año, se manifestarán, en relación a igual número de mujeres que no usen terapia de sustitución hormonal, los siguientes aumentos 0 disminución de patologías.

- siete eventos coronarios más,

- ocho accidentes cardiovasculares más,

- ocho embolias pulmonares más,

- ocho cánceres mamarios invasivos más,

- seis cánceres colorrectales menos, y

- cinco fracturas de caderas menos.

Este estudio sólo consideró un esquema de uso de terapia de estrógenos más progestinas y sus resultados no son necesariamente aplicables a dosis menores de hormonas, a otras formulaciones hormonales orales 0 al uso de hormonas por vía transdérmica. Asimismo, no se distingue el efecto del estrógeno y de la progestina por separado y ello pudiera ser importante por el efecto en particular de las progestinas fundamentalmente en relación a cáncer de mama y enfermedades ateroscleróticas.

Los resultados entregados constituyen la primera información definitiva en que basarse para recomendaciones en el tratamiento de mujeres postmenopáusicas sanas con útero intacto. No se consideraron los riesgos o beneficios de la terapia hormonal de corto plazo para el tratamiento de los síntomas postmenopáusicos. Este esquema de terapia hormonal no debiera ser iniciada o continuada para prevención primaria de enfermedad cardiovascular coronaria y los eventuales hechos positivos relacionados con la disminución de fracturas deben ser sopesados frente a los casos de cáncer mamario y de enfermedad cardiovascular, sobre todo teniendo en consideración la posibilidad de uso de otros agentes terapéuticos para el tratamiento de la osteoporosis.

Finalmente, es necesario correlacionar los resultados del estudio con investigaciones básicas que han reportado la distinta acción que ejercen las progestinas sobre el tejido de la glándula mamaria, acción muy diferente a la ejercida sobre el tejido endometrial donde produce un antagonismo con el efecto proliferativo de los estrógenos disminuyendo el número de mitosis celulares y madurando el tejido a una etapa secretora. En la mama las progesterona produce, in vivo, un efecto proliferante en el tejido mamario durante la fase lútea del ciclo menstrual, como asimismo las elevadas concentraciones plasmáticas de ella en el transcurso del embarazo ayudan a la proliferación mamaria en preparación para la lactancia.

Dra. M. Ruiz F. Dr. Soto, su exposición es muy completa y nos permite apreciar la seriedad de trabajo WHI. Gracias.

A continuación el Dr. Patricio Contreras, se referirá al empleo de las progestinas y cáncer de mama.

Dr. P. Contreras C.: El término abrupto de la rama de la Women's Health Initiative (WHI) que estudió los riesgos y beneficios de la terapia combinada continua con Prempak (estrógenos conjugados equinos $0,625 \mathrm{mg}$ y acetato de medroxiprogesterona $2,5 \mathrm{mg}$ ) en mujeres menopáusicas de 50-79 años, redujo el tiempo de seguimiento de 8 a 5,2 años en promedio. La causa específica de la detención del estudio fue un aumento del riesgo relativo de cáncer mamario invasor en las mujeres que recibían el Prempak y la decisión estuvo a cargo del comité de Seguridad y Monitoreo de Datos del estudio, entidad aparte de los investigadores, los que se mantuvieron ciegos a lo largo del estudio. Lo realmente interesante de este hallazgo es el hecho que había sido predicho en 1998 por Key y Pike, quienes, estudiando los factores de riesgo epidemiológicamente discernibles, establecieron la hipótesis que el riesgo de cáncer mamario aumentaba cuando la mama se exponía a los estrógenos (endógenos o exógenos) y que aumentaba más aún cuando se exponía además, a progesterona o progestinas. La hipótesis, así enunciada, fue paulatinamente obteniendo apoyo en los estudios de la epidemiología de observación. Así, en el Tercer Simposio Lynn Sage de Cáncer de Mama (Octubre 2001), el epidemiólogo Malcolm Pike, PhD de la Universty of Southern California, opinó que el componente progestínico de la terapia combinada representa la causa más prevenible de cáncer mamario en las mujeres norteamericanas y estimó que la THR era responsable de aproximadamente un $10 \%$ de los cánceres mamarios en mujeres postmenopáusicas. Los resultados del WHI serían una demostración experimental que las progestinas, agregadas a los estrógenos, incrementan el riesgo de cáncer mamario más allá del riesgo es- 
perado con estrógenos solos. Así, el aumento de riesgo relativo de cáncer mamario invasor con estrogenoterapia sola se había estimado en un $2,3 \%$ por año de uso, después de 5 años, concentrándose en mujeres con un riesgo natural bajo, como sucede en mujeres delgadas. En el WHI, el aumento observado de riesgo relativo de cáncer mamario invasor fue de $5 \%$ ( $26 \%$ en 5,2 años), más del doble del esperado. En términos de riesgo absoluto, lo anterior se traduce en 38 versus 30 casos de cáncer de mama por cada 10.000 usuarias de THR combinada continua por año. Sin embargo, hubo una alta tasa de abandono de tratamiento. Si se restringe el análisis a las que recibieron por lo menos un $80 \%$ del período estudiado la THR combinada continua, el riesgo relativo se eleva de 1,26 a 1,49 , lo que se traduce en un riesgo absoluto de 44 versus 30 casos cáncer de mama por cada 10.000 usuarias de THR combinada continua por año.

\section{Incidencia del cáncer mamario en el mundo}

Lo más notable es que las tasas de incidencia ajustadas por edad varían unas 6-10 veces en el mundo, desde las observadas en EE.UU. y Europa del Norte a las registradas en Asia y Africa. América Latina y Europa del Sur tienen tasas intermedias entre ambos extremos. Las tasas más altas se dan en EE.UU., con unos 90 casos por cada 100.000 mujeres por año y las más bajas en países asiáticos, como Corea con unos 15 casos por 100.000 . En EE.UU., el riesgo vital de cáncer mamario es de hasta una de cada 8 mujeres. Comparativamente en Chile, si nos guiamos por el hecho que mueren unas 1.000 mujeres al año por esta patología, el riesgo vital sería de 1 por cada 20 mujeres. EI aumento de la expectativa de vida, el sedentarismo creciente y los mayores niveles de ingesta calórica seguramente aumentarán el riesgo de esta neoplasia en nuestro país.

\section{¿Genes o ambiente?}

Estas divergencias tan pronunciadas en las tasas de cáncer mamario no se deberían a influencias genéticas ya que se ha observado que las chinas o japonesas que migran a EE.UU., en una o dos generaciones alcanzan prácticamente las tasas de cáncer mamario del país anfitrión. De los factores ambientales que incidirían en estas diferencias, el sedentarismo y la sobreingesta calórica de los países desarrollados serían la más importantes.

\section{Mecanismos involucrados}

Si revisamos el riesgo de cáncer mamario en 3 poblaciones tipo veremos cómo el riesgo aumenta hasta en 120 veces. El riesgo menor, igual a uno, se observa en la población masculina, que presenta en EE.UU., un riesgo mínimo de cáncer mamario, registrándose sólo 1.500 casos anuales; el riesgo máximo se observa en la contrapartida femenina en ese país, en el cual se registran 180.000 casos anuales (120 veces más). Un riesgo intermedio se observa en la población femenina de países caracterizados por ingestas calóricas moderadas y actividad física elevada, como la de los países asiáticos donde, en una población del tamaño de los EE.UU., se darían sólo 30.000 casos por año (riesgo igual a 20). En síntesis, la presencia de actividad ovárica eleva el riesgo de cáncer mamario en unas 20 veces, mientras que la presencia de actividad ovárica asociada a sedentarismo y sobreingesta calórica, multiplica este riesgo unas 6 veces más. ¿Cómo es que los factores mencionados pueden multiplicar el riesgo de cáncer mamario? Aparentemente, al provocar la aparición y la acentuación del fenómeno de la resistencia insulínica por la obesidad asociada generan un hiperinsulinismo que amplifica la secreción de estrógenos y progesterona ováricos, además de la secreción de testosterona. Así, se ha demostrado un paralelismo entre niveles de progesterona salival en fase lútea, incidencias regionales de cáncer de mama y niveles de ingestión calórica (Jesienska G et al. BMJ 2001; 322: 586). También los niveles de estradiol circulante son mayores notablemente en mujeres inglesas comparados con los de mujeres campesinas en China $(\mathrm{Br} \mathrm{J}$ Cancer 1990; 62: 631. Las diferencias se van acentuando a medida que avanza la edad, siendo máximas después de la menopausia. Además, el hiperinsulinismo provocará un descenso de los niveles circulante de la SHBG, proteína que transporta los estrógenos, determinando un aumento de los niveles biodisponibles en circulación. Por último, los adipocitos mamarios repletos de triglicéridos, aumentan la secreción de citoquinas de tipo I (Interleuquinas 1 y 11, LIF y Oncostatina) y Factor de Necrosis Tumoral alfa, lo que induce mayor aromatización de androstenediona a estrona por los preadipocitos y adipocitos de la mama, creando así un microclima mamario hiperestrogénico.

\section{Resistencia insulínica, obesidad androide y riesgo de cáncer mamario}

En junio de este año, Stoll revisó la relación entre la obesidad androide, la resistencia insulínica 
y el riesgo de cáncer mamario (Int J Obesity 2002; 26: 747). En los países ricos la incidencia de obesidad en mujeres menopáusicas es enorme, siendo de 64\% en las de 50-69 años en EE.UU., según el NAHNES III. Como es bien sabido, la ausencia de hormonas ováricas produce un depósito de grasa por defecto en el compartimento visceral del abdomen en vez del compartimiento fémoro-glúteo. Es responsabilidad del médico combatir la obesidad, el sedentarismo, el tabaquismo y la ingestión significativa de alcohol en la mujer menopáusica para reducir el riesgo de cáncer mamario. Los riesgos de la resistencia insulínica en la mujer menopáusica se pueden resumir en: mayores riesgos de cáncer de mama y colon y mayores riesgos cardiovascular y de diabetes, a los que se suma un envejecimiento acelerado ya que el adipocito repleto secretará menores cantidades de adiponectina (hormona insulino-sensibilizadora y anti-inflamatoria) y mayores cantidades de señales proinflamatorias y protrombóticas que dañan el endotelio vascular (Interleuquina 6, Factor de Necrosis Tumoral alfa, PAl-1, etc.).

\section{Administración de progestinas en la THR y cáncer de mama}

EI WHI nos ha demostrado, consistentemente con los hallazgos observacionales, que las progestinas -si bien protegen al endometrio de una neoplasia- amenazan la mama. Este nuevo conocimiento variará la forma de prescribir THR. Los clínicos evitarán su uso en mujeres histerectomizadas y seguramente reducirán las dosis y la frecuencia de uso de las progestinas. La THR con combinados continuos probablemente será cada día menos frecuente y los clínicos explorarán los beneficios de las progestinoterapias cada tres meses o bien usarán progesterona vaginal o intrauterina) (Progestasert) para actuar directamente sobre el tejido que se pretende proteger. La progesterona micronizada vaginal, en dosis de $100 \mathrm{mg}$ por 12 noches, proporciona una descamación endometrial adecuada y los niveles tisulares de progesterona con esta modalidad son más de 40 veces superiores a los presentes en circulación, permitiendo una reducida exposición mamaria a progesterona. También, la casa Orion se encuentra trabajando en un dispositivo intrauterino pequeño, adecuado al tamaño uterino de las mujeres menopáusicos que libera la mitad de levonorgestrel que el Mirena. Probablemente este nuevo DIU se convierta en una posibilidad atractiva para evitar un cáncer endometrial sin amenazar la mama, ya que protegerá adecuadamente al endometrio, provocará amenorrea y no generará niveles circulantes significativos de levonorgestrel.

Dra. M. Ruiz F.: Gracias: Escucharemos la palabra del Dr. Eugenio Arteaga, sobre los efectos y relaciones cardiovasculares de la Terapia de Reemplazo Hormonal.

Dr. E. Arteaga U.: Es tal el impacto que el estudio WHI ha provocado en el ambiente médico y en la comunidad en general, que es muy probable que a futuro dividamos las informaciones y conductas terapéuticas en "antes y después del estudio WHI".

Si bien el argumento para suspender prematuramente el estudio fue que el número de casos de cáncer de mama invasor detectados en la rama con terapia hormonal de reemplazo (THR) superaron el margen de seguridad prefijado por los investigadores, este hallazgo no fue una novedad. La gran mayoría de los estudios observacionales y todos los meta-análisis sugerían fuertemente que la THR se asociaba a un leve incremento de dicho riesgo, que se hacía evidente después de 5 años de uso y que era proporcional al tiempo de uso de la terapia.

En el caso de las enfermedades cardiovasculares el estudio WHI produjo la mayor sorpresa, al demostrar un aumento de los eventos coronarios, sumado a lo que ya se conocía por los estudios observacionales de un incremento del riesgo de tromboembolismo venoso embolia pulmonar y accidente cerebrovascular.

Los estudios observacionales, en su inmensa mayoría, habían sugerido que la THR reducía el riesgo coronario entre un 30 a $50 \%$. La pregunta es porqué el estudio WHI no encontró este mismo beneficio, y por el contrario demostró un incremento del riesgo de 7 casos de eventos coronarios extra por cada 10.000 pacientes año.

Analizaremos brevemente las razones para esta grave discrepancia entre los estudios epidemiológicos observacionales y el estudio prospectivo $\mathrm{WHI}$.

\section{Mecanismos de protección cardiovascular de la THR}

Se han descrito múltiples mecanismos de la THR, y especialmente de su componente estrogénico, que hacían plausible el beneficio cardiovascular reportado por los estudios observacionales. Entre ellos destacan la mejoría del perfil lipídico, específicamente la elevación de HDL y reducción de LDL y Lp (a), vasodilatación arterial, aumento de la secreción de óxido nítrico y el efecto antioxidante 
de LDL. Además, los estudios en animales demuestran que la THR es capaz de atenuar el desarrollo de ateroesclerosis en modelos experimentales sometidos a dieta aterogénica, situación que también se ha demostrado en arterias carotídeas de mujeres postmenopáusicas tratadas con estradiol puro.

\section{Mecanismos potencialmente dañinos de la THR en el sistema cardiovascular}

A este nivel hay 3 mecanismos importantes. El más conocido es el aumento de los triglicéridos, fenómeno que se ve exclusivamente con la administración oral de estrógenos y que se debe al estímulo de la síntesis de VLDL debido al primer paso hepático. En la mayoría de los casos, este efecto no tiene implicancias clínicas, a excepción de las pacientes que se presentan con hipertrigliceridemia basal como es frecuente en las obesas e insulinorresistentes.

El segundo aspecto negativo es el efecto protrombótico de la THR, predominante -y quizás exclusivo- de la vía oral y que se objetiva en incremento de factores de coagulación, tal como el factor activador de protrombina $(\mathrm{F} 1+2)$, y reducción del factor anticoagulante antitrombina 3 . Si bien existe también estimulo de la fibrinolisis, expresada por reducción del factor PAI-I, y aumento de la capacidad fibrinolítica global (Scarabin PY et al. Arterioscl Thromb Vasc Biol 1997; 17: 3071), el balance final es en pro de la coagulación. Esto se ha objetivado en aumento de 2-3 veces en el riesgo de tromboembolismo venoso y embolia pulmonar, en comparación a los grupos controles (Hulley $S$ et al. JAMA 1998; 280: 605).

En tercer lugar, los estrógenos administrados por vía oral -pero no los que se administran por vía transdérmica- inducen un aumento de la síntesis hepática de la proteína $C$ reactiva (o PCR) (Custman $\mathrm{M}$ et al. Circulation 1999; 100: 717) que es un marcador de inflamación y que se ha descrito como un factor de riesgo de aterosclerosis.

\section{Interpretación de los hallazgos cardiovasculares del WHI}

Como se observa en la figura, el incremento de eventos coronarios en el grupo con THR (expresado como porcentaje del grupo en cada año de tratamiento) fue especialmente notorio en el primer año de terapia, y posteriormente tendió a igualarse con el riesgo del grupo placebo. Un efecto similar de incremento del riesgo de infarto al miocardio solo el primer año con descenso posterior también se observó en el estudio HERS (Hulley S. et al: JAMA
1998; 280: 605) y en el estudio de las Enfermeras (Grodstein F et al: Ann Intern Med 2001; 135: 1).

Este fenómeno puede ser explicado a través del efecto protrombótico y proinflamatorio de la THR oral que se expresó tempranamente en una selección de pacientes de mayor riesgo coronario que desarrollaron precozmente un evento en este territorio. Descartados estos pacientes del estudio desde el momento que sufrían el evento coronario, el riesgo tiende a mantenerse cercano a 1. Además, debemos considerar que el grupo estudiado era mucho más añoso (promedio 62 años) que las pacientes que habitualmente inician THR en nuestro medio y por tanto estos resultados no son enteramente extrapolables a la realidad cotidiana. Además, si uno observa detenidamente la casuística, es difícil asegurar que todas las pacientes estuvieran cardiovascularmente sanas al inicio del estudio, este último debido a la edad avanzada, la alta prevalencia de factores de riesgo cardiovascular y el antecedente de patología coronaria previa en 5\% de ellas, (angina, infartos, bypass coronarios).

Desde el punto de vista conceptual, la placa de ateroma y los eventos coronarios subsecuentes tienen tres componentes principales: el componente de lípidos que se depositan en la pared vascular y forman la placa ateromatosa, los factores inflamatorios que la acompañan y los eventos trombóticos que la pueden complicar. La THR por vía oral, si bien tiene beneficios en el componente lipídico de esta tríada, es procoagulante y proinflamatorio y en definitiva se asoció en el estudio HERS y WHI a incremento temprano de los infartos al miocardio. La terapia transdérmica, en cambio, se asocia a efectos lipídicos menores, pero carece-

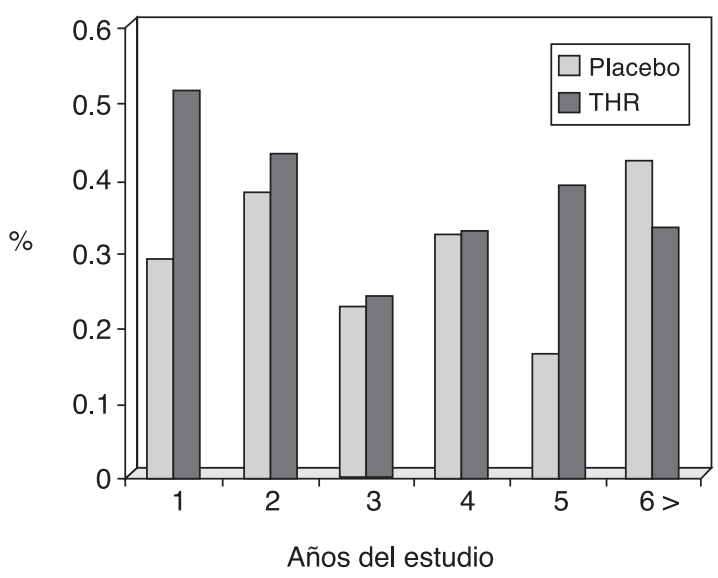

Figura 2. Porcentaje de pacientes que desarrollaron eventos coronarios en cada año del estudio WHI. Jama 2002; 288: 321-333. 
ría de acciones proinflamatorias y protrombóticas, por lo que no se pueden extrapolar los estudios del WHI o HERS a esta modalidad de administración. La pregunta que cabe es, ¿daría lo mismo si se administra THR desde el climaterio temprano, antes que se desarrollen o se extiendan las placas de ateroma, o a mujeres añosas, como en el estudio $\mathrm{WHI}$, en las que probablemente ya existían placas depositadas? La respuesta probable es no, aunque deberemos esperar que otros estudios respondan esta interrogante. Cabe destacar, sin embargo que en mujeres postmenopáusicas con hipercolesterolemia moderada, la administración de $1 \mathrm{mg}$ de estradiol oral se asoció a disminución del grosor de la íntima-media, en comparación al grupo placebo que mostró incremento del mismo, lo que sugiere fuertemente un efecto antiaterosclerótico de los estrógenos puros (Hodis HN, et al. Ann Intern Med 2001; 135: 939).

En conclusión, los resultados de los estudios HERS y WHI no han demostrado protección cardiovascular secundaria ni primaria. Por lo contundente de estos hallazgos $-y$ a menos que se publiquen otros trabajos que contradigan sus resultados- se debe eliminar la indicación de THR para la prevención cardiovascular. Respecto al posible daño que pudiera ejercer la THR en el sistema vascular, a excepción del fenómeno tromboembólico, que es en general de muy baja incidencia, creemos que el riesgo coronario demostrado en el estudio $\mathrm{WHI}$ no es aplicable a pacientes "sanas y sintomáticas" que inician THR en la perimenopausia. En aquellas pacientes con factores de riesgo coronario, sin embargo, es prudente utilizar la menor dosis de estrógeno que sea efectiva o preferir la vía no oral.

Deberemos estar atentos a la rama del estudio WHI que utiliza sólo estrógenos conjugados equinos $0,625 \mathrm{mg}$ y que aún continúa activa, y al estudio WISDOM de los ingleses, que en contraste a lo acontecido en el estudio WHI, que suspendió la rama de THR combinada, han decidido mantener su protocolo y nos informarán a futuro del riesgo cardiovascular observado en esta cohorte

Dra. M. Ruiz F.: El completo análisis, parece demostrar los efectos no deseables de ésta terapia particularmente en tromboembolismos y enfermedades coronarias. Muchas gracias.

Finaliza esta presentación una destacada especialista en mama como la Dra. Raquel Herrera. Tiene la palabra.

Dra. R. Herrera: La revisión de la evidencia acumulada en las últimas décadas en relación al papel de los Estrógenos y de la Progesterona en el aumento del riesgo del cáncer de mama, hace que los resultados publicados por el Women's Health Iniciative en este sentido no sean sorprendentes.

Se conoce de antiguo el papel de las hormonas ováricas en la promoción e incluso en la génesis del cáncer de mama. Dicho rol se fundamenta en la frecuencia 100 veces mayor de esta neoplasia en la mujer que en el hombre, en el aumento del riesgo que se observa en mujeres con menarquia precoz y/o menopausia tardía; así como en el importante hecho de que la curva logarítmica de las tasas de incidencia respecto a la edad se desacelera en relación al advenimiento de la menopausia (Pike Epidemiologic Reviews, 1993).

Se ha propuesto la hipótesis de que una exposición más prolongada a ciclos ovulatorios significan un aumento del riesgo de cáncer de mama. Esto se basa en el hecho comprobado de que el número de mitosis se duplica o triplica durante la fase lútea del ciclo comparado con la fase proliferativa, lo que pone en el tapete la influencia de la Progesterona en este proceso.

Este hecho se ve confirmado por un riesgo menor en mujeres con infertilidad por anovulación y en mujeres obesas premenopáusicas, que también presentan con mayor frecuencia ciclos anovulatorios. Apoyan también el papel de los Estrógenos endógenos en el cáncer de mama los efectos multifactoriales del embarazo en el riesgo, como también la asociación entre niveles circulantes de Estrógenos más altos en mujeres postmenopáusicas (como por ejemplo obesas), y una mayor probabilidad de cáncer de mama.

El cuerpo de evidencias actualmente disponible proviene de:

- Estudios de la influencia de los estrógenos y progesterona en la proliferación del epitelio mamario.

- Del estudio de los niveles plasmáticos de estradiol y su relación con el riesgo de cáncer de mama.

- De la relación de marcadores clínicos de exposición a estrógenos y su correlación directa con el riesgo de cáncer de mama (densidad ósea, densidad radiológica de la mama).

- De estudios epidemiológicos de cohorte y de casos y controles que muestran un aumento del riesgo con el uso de Tratamiento de Sustitución Hormonal con Estrógenos solos y un incremento del riesgo cuando se emplean Estrógenos + Progestinas.

Se analizarán en forma breve estos antecedentes.

En relación a la Terapia de Sustitución Hormonal (TSH) en el climaterio, ella ha sido extensamen- 
te investigada. Existen 6 meta-análisis y un reanálisis combinado de 51 estudios epidemiológicos sobre la materia. (Collaborative Group on Hormonal Factors in Breast Cancer. The Lancet, 1997).

En Estados Unidos y Europa la incidencia acumulativa de cáncer de mama es muy alta, siendo, en mujeres $\sin \mathrm{TSH}$, de 18 por cada 1.000 a los 50 años llegando a 77 por cada 1000 a los 75 años.

Los resultados de este último trabajo indican que la prolongación del TSH por más de 5 años aumenta levemente el riesgo relativo (RR) de cáncer mamario y que este aumento desaparece después de 5 años de suspendido el tratamiento. El RR como TSH es de 1.023 por año, un aumento discreto de $2,3 \%$ anual, cifra muy similar a la que se observa con el atraso de la ocurrencia de la menopausia. En este estudio (Estados Unidos y Europa), las cifras muestran que entre los 50 y los 70 años la incidencia acumulativa de cáncer de mama es de 45 por cada 1.000 mujeres en aquellas que no usan TSH (va de 18 por cada 1.000 mujeres a los 50 años a 63 por cada 1.000 mujeres a los 70 años). En las usuarias, de acuerdo al 2,3\% de aumento del riesgo anual hay dos, seis y doce casos más por cada 1.000 mujeres con 5,10 y 15 años de uso.

Un estudio de casos y controles (Magnusson. Int J Cancer, 1999), que incluyó 3345 mujeres con cánceres infiltrantes de la mama, versus 3454 controles de edades similares, mostró una asociación positiva con el uso de TSH que incluía Estrógenos + Progestinas (Acetato de Medroxiprogesterona, o derivados de la Testosterona como: Noretisterona Acetato, Levonorgestrel o Linestrenol). Hubo una tendencia al aumento del riesgo a medida que se prolongaba el tratamiento. Para las mujeres tratadas durante al menos 10 años el riesgo relativo aumentó a $2.43 ; 95 \% \mathrm{Cl}, 1.79-3,30$, siendo los resultados estadísticamente significativos solo en las mujeres con bajo Indice de Masa Corporal. El aumento del riesgo era especialmente pronunciado cuando el TSH empleado era continuo.

Otro estudio, esta vez de cohorte (Schairer. Jama, 2000) involucró 46355 mujeres postmenopáusicas seguidas desde 1980 hasta 1995, grupo dentro del cual se presentaron un total de 2082 caos de cáncer de mama. El estudio estaba diseñado para determinar si el riesgo asociado con Tratamiento de sustitución hormonal bajo el régimen de Estrógenos-Progestinas era mayor que aquel asociado con el empleo de Estrógenos aislados.

Sus resultados sugieren que el riesgo de cáncer de mama es mayor en los regímenes de TSH con Estrógenos-Progestinas, lo cual es válido para mujeres con Indice de Masa Corporal (BMI) de 24,4 $\mathrm{kg} / \mathrm{m}^{2}$ o menos. El riesgo en las mujeres obesas no aumentó con ninguna de las dos modalidades de tratamiento.

La más reciente de las publicaciones basada en los datos del Estudio de las Enfermeras (Colditz. Am J Epidemiol, 2000) sigue una cohorte de 58.520 mujeres desde 1980 hasta 1994. En este período 1.861 casos de cáncer de mama infiltrante fueron pesquisados.

El nuevo análisis de los datos muestra que el TSH con Estrógenos solos de los 50 a los 60 años aumentó el riesgo de cáncer de mama en un $23 \%$ (Cl: $6 \%, 42 \%$ ) a la edad de 70 años comparado con no usuarias de Terapia de Sustitución Hormonal. Diez años de uso de Estrógenos + Progestinas aumentaron el riesgo a los 70 años en un $67 \%(\mathrm{Cl}$ : $18 \%, 136 \%)$.

El trabajo concluye que el uso de TSH por 10 años con Estrógenos solos aumenta significativamente el riesgo de cáncer de mama, y que la adición de Progestinas al tratamiento eleva aún más este riesgo.

Un trabajo basado en casos y controles (Ross. J Natl Cancer Inst, 2000) incluye 1897 casos de cáncer de mama versus 1637 controles. Se determinó el riesgo en relación a diferentes regímenes de sustitución hormonal. Sus resultados muestran un aumento del $10 \%$ del riesgo por cada 5 años de uso de TSH $(\mathrm{OR}=1,10 ; 95 \% \mathrm{Cl}=1,02-1,18)$. El riesgo fue sustancialmente mayor para los tratamientos que incluían Progestinas $(\mathrm{OR}=1,24 ; 95 \%$ $\mathrm{Cl}=1,07-1,45)$ que para aquellos con Estrógenos solos $(\mathrm{OR}=1,06 ; 95 \% \mathrm{Cl}=0,97-1,15)$.

Los autores concluyen que hay evidencia fuerte en el sentido de que la adición de Progestinas a la Terapia de Sustitución Hormonal incrementa en forma marcada el riesgo de cáncer de mama con respecto al uso de Estrógenos en forma exclusiva.

Como ya se mencionó, otra vertiente importante en el análisis del riesgo de cáncer de mama es la investigación de posibles marcadores clínicos de exposición a Estrógenos.

Investigaciones relativamente recientes han mostrado una directa relación entre niveles plasmáticos de estrógenos medidos en un solo punto en el tiempo y el riesgo de cáncer de mama. En varios estudios se demuestra que mujeres en las cuales se desarrolla ulteriormente un cáncer de mama tenían mayores concentraciones plasmáticas de estradiol libre.

Estas determinaciones se hicieron en un estudio prospectivo de fracturas osteoporóticas (Cauley 
Jama, 1996) que indica además que la densidad ósea en la muñeca, cadera y columna tiene una fuerte correlación directa y estadísticamente significativa con el riesgo de cáncer de mama.

Se ha propuesto la densidad ósea como marcador de exposición acumulativa a los Estrógenos en la mujer.

En el estudio de Framingham (Zhang NEJM, 1997) se determinó la densidad ósea de 1373 mujeres que fueron seguidas desde 1967 hasta 1993. Se produjeron 91 cánceres de mama, de los cuales la incidencia por 1.000 personas-año aumentó de 2,0 en el cuartil con densidad ósea más baja, a 2,6; 2,7 y 7,0 en los cuartiles $2^{\circ}$, $3^{\circ}$ y más alto respectivamente. Después de los ajustes de acuerdo a la edad y otros factores de confusión, los riesgos relativos fueron de 1,$0 ; 1,3$; y 3,5 desde el cuartil más bajo al más alto.

Los autores concluyen que el cuartil de densidad ósea más alto tiene un riesgo aumentado de cáncer de mama, lo que podría deberse a una exposición acumulativa mayor a los Estrógenos.

Otro marcador clínico de exposición a estrógenos es la densidad radiológica de la mama. Su apariencia mamográfica varía de una mujer a otra dependiendo de las cantidades relativas de grasa y tejido fibroepitelial que la componen. Numerosos estudios reportan una asociación entre los distintos patterns mamográficos y el riesgo de cáncer de mama. A mayor densidad mayor riesgo.

Un estudio (Boyd J Natl Cancer Inst, 1995) efectuó una clasificación cuantitativa de la densidad mamográfica de 354 casos de cáncer de mama y 354 controles de una cohorte de 45.000 mujeres asignadas a un Programa de Pesquisa Precoz en el Canadian National Breast Cancer Screening Study. Se estudiaron mujeres de $40-49$ y 50-59 años. Se encontraron aumentos estadísticamente significativos del riesgo asociados a los aumentos en la densidad radiológica, tanto con la estimación hecha por radiólogos, como en la asistida computacionalmente.

Las mujeres con mamas radiológicamente densas tienen niveles séricos más altos de Estrógenos que las mujeres con mamas adiposas. Más aún el Tratamiento de Sustitución Hormonal frena o detiene la involución adiposa fisiológica de la glándula en un 75 a $90 \%$ de los casos y la revierte en el 10 a 25\% (Gairard Genesis, 1998. Meyer J Radiol, 2001).

Una importante investigación del efecto de los Estrógenos y la Progesterona sobre la proliferación del epitelio mamario (Hofseth, Haslam. J Clin Endocrinol Metabol, 1999) se realizó en 86 casos de biopsias mamarias que resultaron ser benignas. Se buscaba examinar los efectos del Tratamiento de Sustitución Hormonal con Estrógenos solos o con Estrógenos + Progestinas (Acetato de Medroxiprogesterona).

Se analizaron las muestras con antígeno antiproliferativo nuclear (anti-PCNA) y anticuerpos ki67 para medir los niveles relativos de proliferación celular. También se determinaron la densidad del epitelio y el número de receptores hormonales.

Comparadas con mujeres que no habían recibido TSH las tratadas con Estrógenos solos tenían niveles de proliferación celular significativamente mayores. Estos índices eran aún mayores en mujeres sometidas a TSH con Estrógenos + Progestinas.

La evidencia recién descrita antecede y se suma a la proporcionada por el estudio WHI. Ella aconseja revisar la liberalidad con que se indicaban tratamientos hormonales de sustitución y restringirlos a indicaciones más acotadas a cada caso. Más aún teniendo en cuenta que la protección cardiovascular parece ser muy inferior a la que se suponía, y que tanto para ésta como para la prevención de la osteoporosis hay otras opciones.

Dra. M. Ruiz F.: Muy interesante la presentación de la Dra. Todo esto nos enseña a ser cautelosos, analizando caso a caso cada paciente en particular antes de suscribir acciones de reemplazo hormonal a todas las pacientes en la perimenopausia y menopausia. Agradezco la brillante participación de los componentes de esta Mesa y el entusiasmo del público en escucharnos y participar en estas inquietudes. Muchas gracias. 